\title{
Multiwavelength variability of the radio quasar J2042+7508
}

\section{S. Zola*}

Astronomical Observatory, Jagiellonian University, ul. Orla 171, 30-244 Krakow, Poland

Mt. Suhora Observatory, Pedagogical University, ul. Podchorazych 2, 30-084, Krakow, Poland

E-mail: szola@oa.uj.edu.pl

\section{A. Kuzmicz, M. Jamrozy, G. Bhatta, B. Debski, T. Kundera, D. Koziel-Wierzbowska} Astronomical Observatory, Jagiellonian University, ul. Orla 171, 30-244 Krakow, Poland

\section{Winiarski, W. Ogloza, M. Drozdz, M. Siwak}

Mt. Suhora Observatory, Pedagogical University, ul. Podchorazych 2, 30-084, Krakow, Poland

In this paper, we present our results of study on the long term multiwavelength variability properties of the quasar $\mathrm{J} 2042+7508(4 \mathrm{C}+74.26)$ - a giant radio source located at the redshift of 0.104 . This source exhibits interesting emission and structural properties when observed in various wavelengths, including X-ray, optical and radio frequencies. Therefore, exploring these properties through multifrequency variability studies presents a great importance to our understanding of the evolution of quasars and radio-loud unification schemes.

We found a trend of anticorrelation with time lag of about three months between optical and radio light curves. A weak correlation with a longer time lag of about 230 days might also exist. Using the structure function method, applied to our six years long, optical data, we arrived at a conclusion that the quasar variability with amplitude of about 0.3 magnitude, is likely caused by an accretion disk instability.

SALT Science Conference 2015 -SSC2015-

1-5 June, 2015

Stellenbosch Institute of Advanced Study, South Africa

\footnotetext{
${ }^{*}$ Speaker.
} 


\section{Overall nature of the quasar $\mathrm{J} 2042+7508$}

\subsection{Properties of the radio structure}

The radio quasar $\mathrm{J} 2042+7508$ (alternate name $4 \mathrm{C}+74.26$ ) was disclosed by [18]. This powerful FRII-type ([8]) double-lobe radio source (see Fig.1) is located at R.A.(J2000.0) $20^{\mathrm{h}} 42^{\mathrm{m}} 37 \mathrm{~s} .3$ and DEC.(J2000.0) $+75^{\circ} 08^{\prime} 02^{\prime \prime}$. 5 . Its angular size is about $10^{\prime}$ which for the object redshift of $\mathrm{z}=0.104$ gives the projected linear size of $1.14 \mathrm{Mpc}$ (when $\mathrm{H}_{0}=71 \mathrm{~km} \mathrm{~s}^{-1} \mathrm{Mpc}^{-1}$ is adopted). [19] presented a sensitive $1.5 \mathrm{GHz}$ VLA radio map which shows a large scale $(\sim 350 \mathrm{kpc})$ one sided and south directed radio jet.

[14] estimated the following parameters of the giant radio source: its total and core luminosity at $1.4 \mathrm{GHz}$ is $\log \left(\mathrm{P}_{\text {tot }}\right)=25.67 \mathrm{WHz}^{-1}$ and $\log \left(\mathrm{P}_{\text {core }}\right)=24.72 \mathrm{WHz}^{-1}$, respectively. Furthermore, they obtained additional parameters of the radio structure: the bending angle, which is the complement of the angle between the lines connecting the lobes with the core, to be about 7.1 , the arm-length-ratio (the ratio of distances between the core and the hotspots) close to 1.03.

The hotspot on the southern side is the most-X-ray-luminous hotspot known at low redshift

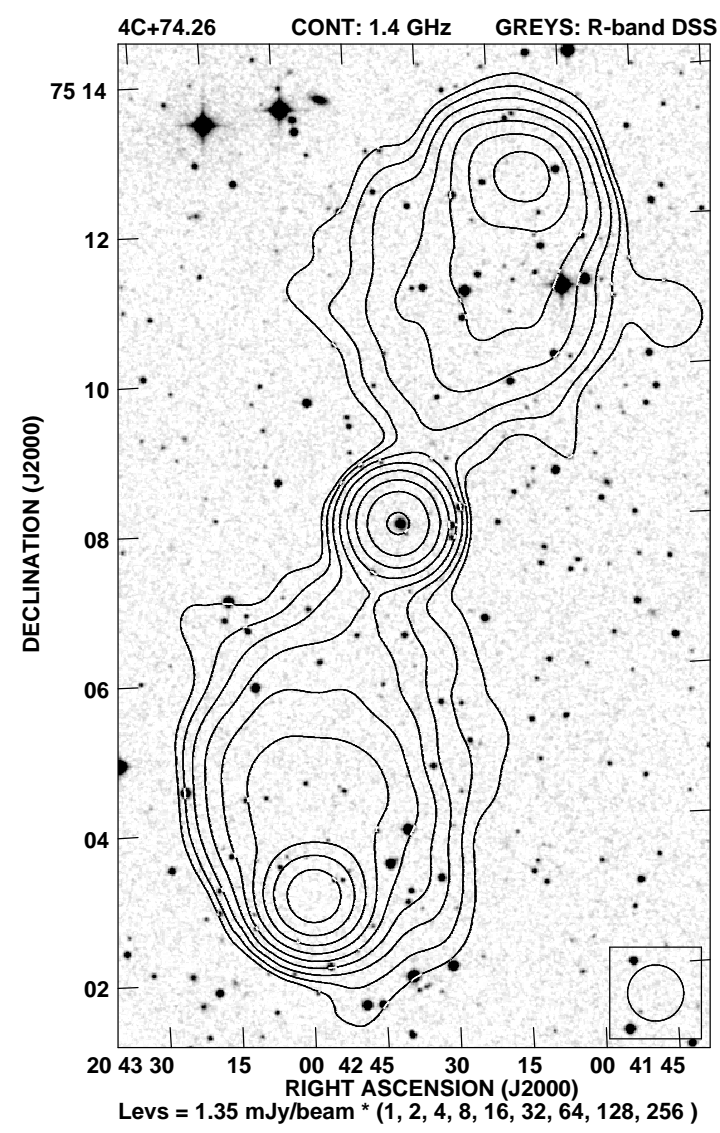

Figure 1: J2042+7508 - radio intensity contours at $1.4 \mathrm{GHz}$ from the NRAO VLA Sky Survey ([5]) overlayed on the optical R-band DSS image. The radio beam size is marked by a circle in the bottom-right corner. 
Table 1: Flux densities of $\mathrm{J} 2042+7508$.

\begin{tabular}{|c|c|c|c|c|c|}
\hline \multicolumn{3}{|c|}{ Radio core } & \multicolumn{3}{|c|}{ Total source } \\
\hline $\begin{array}{l}\text { Freq. } \\
(\mathrm{MHz})\end{array}$ & $\begin{array}{c}\text { Flux \& err } \\
(\mathrm{Jy})\end{array}$ & Ref. & $\begin{array}{l}\text { Freq. } \\
(\mathrm{MHz})\end{array}$ & $\begin{array}{c}\text { Flux \& err } \\
(\mathrm{Jy})\end{array}$ & Ref. \\
\hline 327 & $0.07 \pm 0.006$ & [16] & 38 & $30 \pm 9$ & [18] \\
\hline 599 & $0.174 \pm 0.009$ & [12] & 151 & $9.9 \pm 1.0$ & [18] \\
\hline 1407 & $0.18 \pm 0.02$ & {$[18]$} & 408 & $4.0 \pm 0.1$ & [18] \\
\hline 1460 & $0.184 \pm 0.003$ & [16] & 599 & $4.078 \pm 0.2$ & [12] \\
\hline 1666 & $0.24 \pm 0.02$ & [18] & 1407 & $1.63 \pm 0.08$ & [18] \\
\hline 2695 & $0.34 \pm 0.1$ & [18] & 2695 & $1.1 \pm 0.2$ & [18] \\
\hline 4880 & $0.328 \pm 0.001$ & [16] & 4995 & $0.81 \pm 0.2$ & [18] \\
\hline 4995 & $0.42 \pm 0.1$ & {$[18]$} & 10000 & $0.632 \pm 0.009$ & [21] \\
\hline 4995 & $0.37 \pm 0.1$ & {$[18]$} & & & \\
\hline 4995 & $0.31 \pm 0.1$ & {$[18]$} & & & \\
\hline 4816 & $0.250 \pm 0.013$ & [12] & & & \\
\hline 8400 & $0.333 \pm 0.001$ & {$[16]$} & & & \\
\hline 10000 & $0.300 \pm 0.003$ & {$[21]$} & & & \\
\hline 14900 & $0.306 \pm 0.002$ & [16] & & & \\
\hline 270000 & $0.055 \pm 0.02$ & [18] & & & \\
\hline
\end{tabular}

([7]). It is also significantly brighter than that on the opposite side at radio wavelength. The lobe flux-density ratio of $4 C+74.26$ is 2.69 . The inclination angle, defined as the angle between the jet axis and the line of sight, is $61^{\circ}$. The $10 \mathrm{GHz}$ polarimetric map ([21]) shows that the magnetic field is parallel to the presumed jet towards the lobes, and follows the general trend found in FRIItype radio galaxies that they are circumferential towards the peripheries and nearly orthogonal to the source axis near the hotspots. The southern hotspot is strongly polarized. [12] estimated the magnetic field strength within the radio lobes at 0.17 and $0.20 \mathrm{nT}$ for the northern and southern lobe, respectively. These values are typical of FRII-type extended radio lobes. The spectral index estimated by [21] between 0.15 and $10 \mathrm{GHz}$ of the northern and southern lobe is 0.74 and 0.78 , respectively. [16] presented a high resolution, $5 \mathrm{GHz}$ Very Long Baseline Interferometry map of the core of the quasar. The map shows one-sided parsec-scale jet that is well aligned with the large-scale jet. There was no evidence for a counter-jet. Assuming that the asymmetry of the parsec-scale jet is attributed to Doppler boosting, the axis of the nuclear jet must be $\lesssim 49^{\circ}$ from the line of sight. The total flux density of the core at $5 \mathrm{GHz}$ decreased from 0.42 to 0.31 Jy between 1986 and 1988 ([18]; see also Table 1). In addition, the estimate of $0.25 \mathrm{Jy}$ at $4.8 \mathrm{GHz}$ in 2000 made by [12] is consistent with a strongly variable radio core. The radio variability is also confirmed by monitoring performed at $15 \mathrm{GHz}$ with the 40-m telescope at the Owens Valley Radio Observatory (OVRO; [17]).

\subsection{Optical and X-ray data}

The optical image of $4 C+74.26$ shows that the central source is surrounded by extended, low-brightness emission. The absolute optical magnitudes of the quasar are $\mathrm{M}_{\mathrm{V}} \sim-24.7^{\mathrm{m}}$ and $\mathrm{M}_{\mathrm{B}} \sim-24.5^{\mathrm{m}}$ and its bolometric luminosity is around $2 \times 10^{46} \mathrm{erg} \mathrm{s}^{-1}$ ([24]). This makes it a lowluminosity quasar. [24] estimated the quasar's black hole mass of $4 \times 10^{9} \mathrm{M}_{\odot}$ and Eddington ratio of $\sim 0.04$. [18] showed that the spectrum contains a broad $\mathrm{H}_{\alpha}$ line typical of quasars. The FWHM 
width of the $\mathrm{H}_{\beta}$ line is about $7500 \mathrm{~km} \mathrm{~s}^{-1}$. [20] discovered large variations in both the degree and position angle of polarization across the broad $\mathrm{H}_{\alpha}$ line. The position angle is not precisely orthogonal to that of the radio jet, suggesting that the scattering outflow is either inhomogeneous, or somewhat misaligned with the radio structure. In addition, the polarized feature is redshifted by $\sim 2000 \mathrm{~km} \mathrm{~s}^{-1}$ relative to its median wavelength in total flux. Such a nature of the polarized flux can be explained by scattering in a high-speed outflow which, if it is directed along the axis of the radio jet, must have a velocity of $\gtrsim 5000 \mathrm{~km} \mathrm{~s}^{-1}$ to match the observed redshift.

[4] presented an X-ray study of the quasar 4C +74.26 made by the Advanced Satellite for Cosmology and Astrophysics (ASCA). The ASCA spectrum reveals indications of three ingredients, i.e. a reflection component, a neutral iron line and a warm absorber. The underlying power law has a photon index of $\Gamma=2.03 \pm 0.09$. The soft X-ray flux in 1996 was nearly twice as high as that found in the Roentgen Satellite (ROSAT) observations in 1993 but without apparent spectral changes. The X-ray data support the picture deduced from previous radio observations that $4 \mathrm{C}+74.26$ is seen under a relatively large angle $\left(\sim 45^{\circ}\right)$. [2] presented an evidence of a broad ionized $\mathrm{Fe} \mathrm{K}_{\alpha}$ line in the XMM-Newton spectrum. This was the first indication that the innermost regions of the accretion flow in broad line radio galaxies contain thin, radiatively efficient disks. The $\mathrm{Fe} \mathrm{K}_{\alpha}$ line profile gives an inclination angle of $\sim 40^{\circ}$. This is consistent with the radio limit given above. [15] reported on a 90 ks Suzaku detection. The source brightened by about $20 \%$ during the period of observations. They saw the evidence of spectral hardening as the count rate increased. They concluded that the inner AGN disk is either missing or not strongly illuminated, and suggested that the latter scenario may occur if the power-law source is located high above the disk, or if the emission is beamed away from the disk.

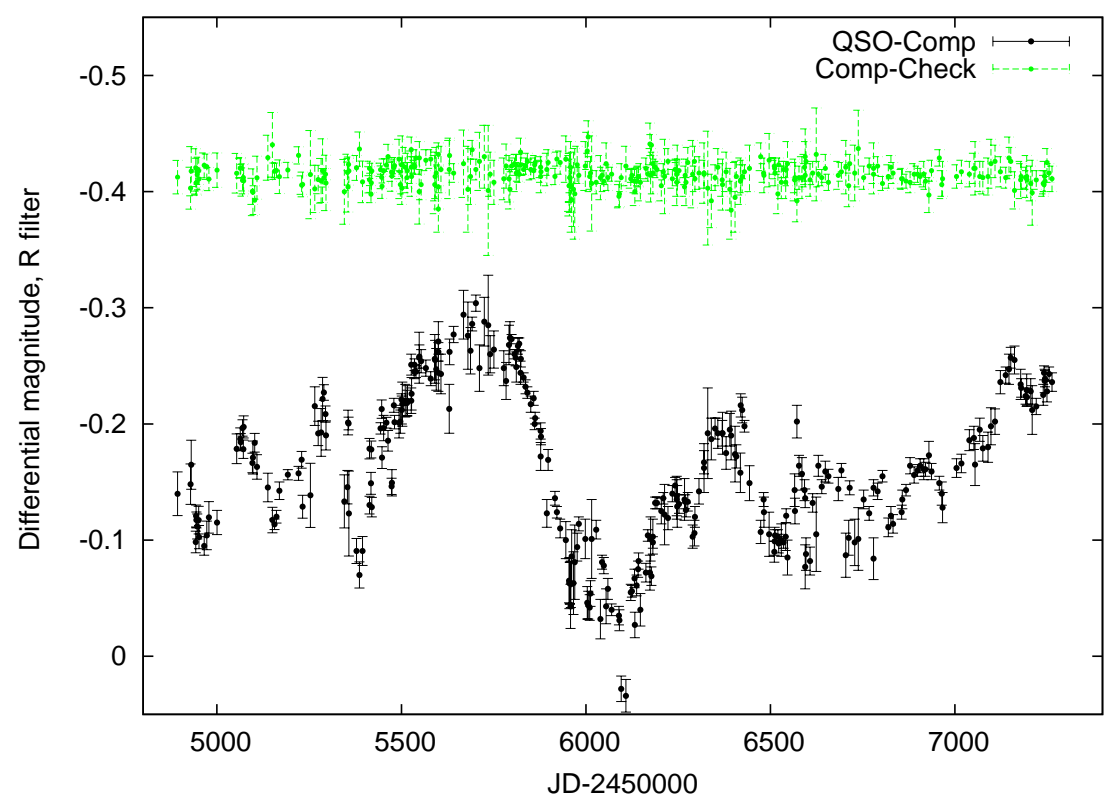

Figure 2: J2042+7508 - optical R-band light curve (dark symbols). Green symbols represent magnitude differences between the comparison and the check stars. 
The source is not a strong gamma-ray emitter and [10] gave its upper limits based on the Fermi Gamma-ray Space Telescope observations: the integrated photon flux above $100 \mathrm{MeV}$ $\mathrm{F}_{>0.1 \mathrm{GeV}}<11 \times 10^{-9} \mathrm{ph} \mathrm{cm}^{-2} \mathrm{~s}^{-1}$, the $\gamma$-ray flux $\left[v \mathrm{~F}_{v}\right]_{0.1-10 \mathrm{GeV}}<4.7 \times 10^{-12} \mathrm{erg} \mathrm{cm}^{-2} \mathrm{~s}^{-1}$, and the corresponding $\gamma$-ray luminosity $\log \left(\mathrm{L}_{\gamma}\right)<44.1 \mathrm{erg} \mathrm{s}^{-1}$.

\section{Analysis of multiwavelength variability of $\mathbf{J 2 0 4 2}+7508$}

\subsection{Optical monitoring}

We have undertaken optical observations of a sample of eight radio quasars in 2009. Among them, J2042 +7508 , with a declination of about $75^{\circ}$, can be observed throughout the entire year at sites located in Poland. The data have been primarily gathered at the Mt. Suhora Observatory of the Pedagogical University in Krakow. The 60-cm telescope equipped with an Apogee CCD, mounted in the prime focus and a set of UBVRI filters (Bessell's specification [3]) was used. Some complementary data have been gathered at the Astronomical Observatory of the Jagiellonian University with the 50-cm telescope equipped with an Andor CCD and a similar set of wide band filters. A series of $\mathrm{R}$ filter images of a target has been obtained every few nights along with calibration images (bias, dark and flatfield, usually on sky). On some occasions also multicolor data (BVRI) were taken. The same comparison (GSC0103002595) and check (GSC0103002574) stars have been used during the entire observing campaign. To achieve as uniform as possible results, despite of several observers being engaged in this project, the reduction of CCD frames was done by the same person. The R filter data gather of J2042+7508 since early 2009 till August 2015 are shown in Fig. 2. The light curve as magnitude differences with respect to GSC0103002595 and GSC0103002574 are plotted in black symbols, while magnitude differences between check and comparison stars are shown as green symbols.

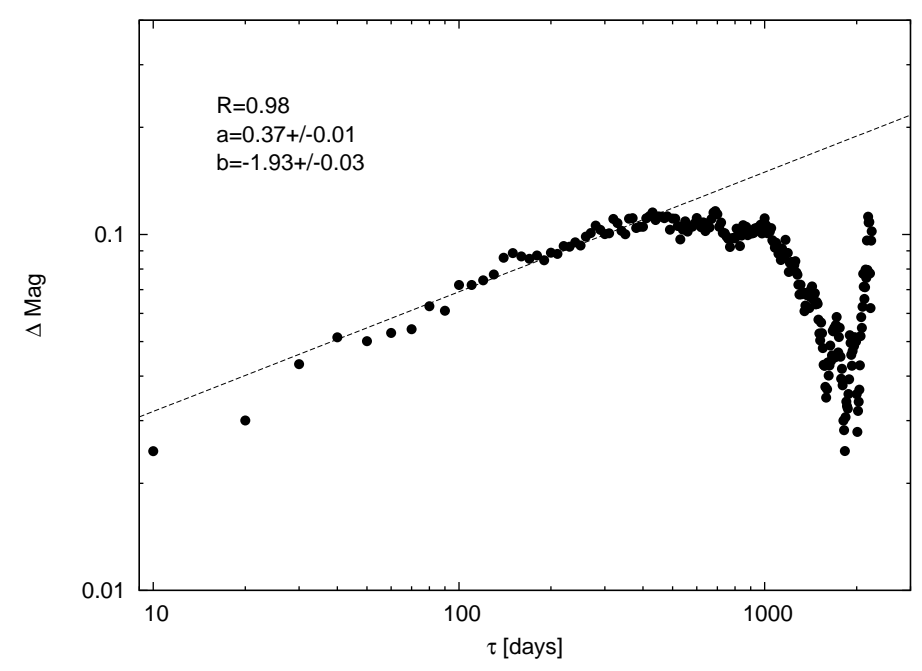

Figure 3: Structure function of $\mathrm{J} 2042+7508$ with a linear fit (dashed line). The parameters of the fit are given in the upper-left corner.

Information from time-series data can be extracted by different methods. These most often used are: Fourier power spectrum, autocorrelation function and structure function (SF). The SF 

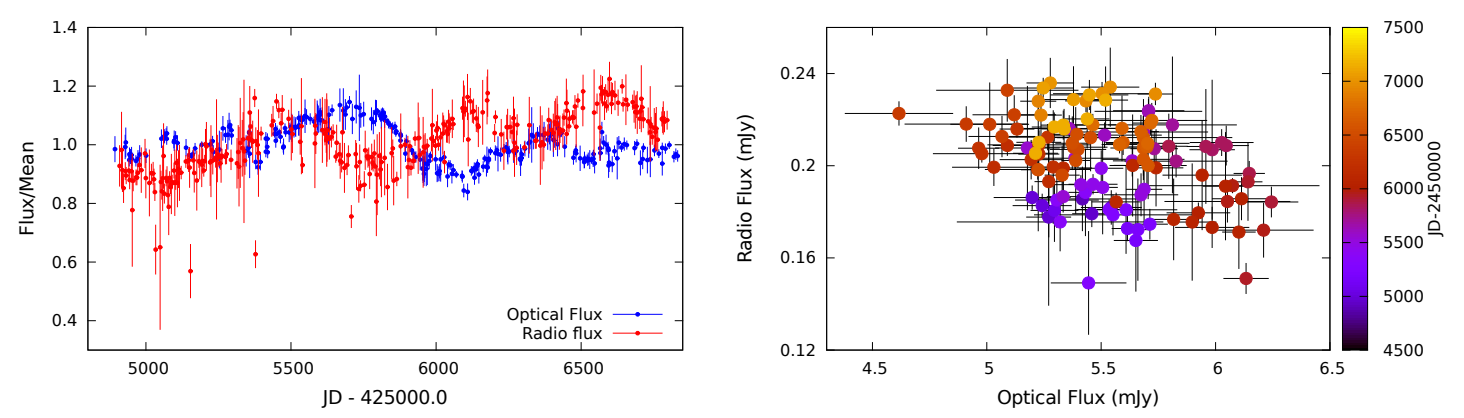

Figure 4: Left panel: optical (blue) and radio (red) light curves of J2042+7508 normalized by their corresponding means. Right panel: Radio flux densities plotted against simultaneous optical fluxes. The time of observations is shown by the color bar.

technique has been widely used to analyze the light curves of quasars. It describes a distribution of variability by measuring the mean value of variation magnitudes separated by a given time interval $\tau$ (the full definition can be found in e.g. [22]). In the case of using the SF method, no information about physical processes can be inferred unless there is a model from which a theoretical SF could be calculated. Only then, by comparing the shapes of the two, one can draw a conclusion about the nature of a phenomenon. Several models has been computed to explain the cause of observed optical variability in quasars: accretion disk instabilities [11], supernovae and starburst processes [1], gravitational microlensing [9]. We applied the SF method to J2042+7508 to reveal its properties from our 6 years long optical data. The calculated shape of SF is shown in Fig. 3. We obtained the SF slope value of $0.37 \pm 0.01$. This indicates, that the optical variability could likely be due to the disk instabilities, caused by a non uniform accretion rate. The time scales, predicted by the model, would be of order of days - for the most inner stable orbit, to years - if they occur in the outer parts of an accretion disk.

\subsection{Cross-correlation of radio, optical and X-ray data}

The optical and radio observations of J2042+7508 were gathered during almost the same period of time. This enables us to check a possible correlation between the light curves in these two wavelengths. Interestingly, a trend of anticorrelation between them can be clearly seen in Fig. 4 - left panel, which presents both light curves normalized by their corresponding means, and also from Fig. 4 - right panel, which shows a plot of simultaneous radio fluxes against optical ones. The correlation between them is further analyzed more robustly by estimating the discrete correlation function (DCF) as described in [6]. This function measures the degree of correlation between two light curves as a function of time lag $\tau$, the shift between the light curves in time. Since DCF within this method is not normalized, the normalization suggested by [23] was applied so that the DCF value equal to 1 indicates completely correlated data, -1 anticorrelated, and a value of 0 indicates no correlation.

The resulting DCF for J2042+7508 is shown in Fig. 5. The 90\%-significance-contour level (shown by dotted curves) estimated by 10000 random pairs of simulated light curves of white noise is also plotted. While computing the DCF, the length of the time lag was limited to about half of 


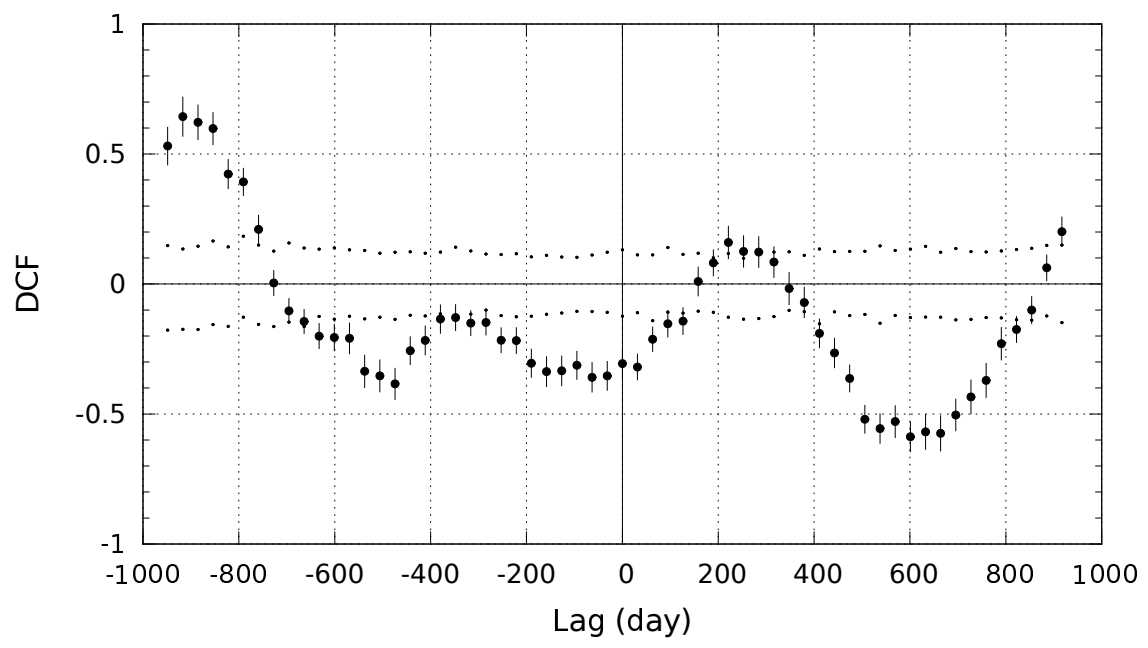

Figure 5: Discrete correlation function for J2042+7508 as the function of time lag in days. The positive lag here means high energy emission leading the lower one. The dotted curves show the $90 \%$ confidence level.

the duration of observations because the DCF estimation involves less number of observation as the time lag becomes larger, making the correlation value less significant.

As it can be seen in Fig. 5, on the positive upper part (correlation), there is one very prominent peak at $\tau=-900$ days and the value of $\mathrm{DCF}=0.67$. There is also another one, at $\tau=230$ days but with very low DCF, barely exceeding the $90 \%$ significance level line. Despite the former peak showing high statistical confidence its time lag is long, close to the considered lag's edge. Therefore, its reliability is doubtful as this result could be spurious due to the correlation found in subsets of data. Only data of significantly longer duration can confirm the presence of correlation with such a long time lag. On the other hand, the correlation with $\tau=230$ days, indicated by the latter peak, though barely significant, looks visually very convincing as seen in the left panel of Fig. 6. Again, only when a longer run is analyzed and the correlation remains present, will it be possible to draw firm conclusions about its nature.

There are three statistically significant peaks in the bottom part (anticorrelations) of Fig. 5: two at longer time lags of $\tau=-500$ and 600 days and another one with smaller value among found peaks at $\tau=-87$ days. We believe that the results for two anticorrelations for longer time lags may not be quite reliable for the data sets with current length as the DCF estimation involves a smaller section of the curves as presented in Fig. 6 - right panel. Using the same DCF method, we also performed cross-correlation of the optical and X-ray data taken from the Swift/Burst Alert Telescope (BAT) database ([13]). We did not find significant correlations between these two bands.

\section{Conclusions}

We analyzed photometric observations of $\mathrm{J} 2042+7508$ taken in the wide band R filter from 2009 to 2015. The structure function of the observed light curve was calculated. Based on the SF properties, we confirm our earlier conclusion ([25]) that the optical variability of the quasar is 

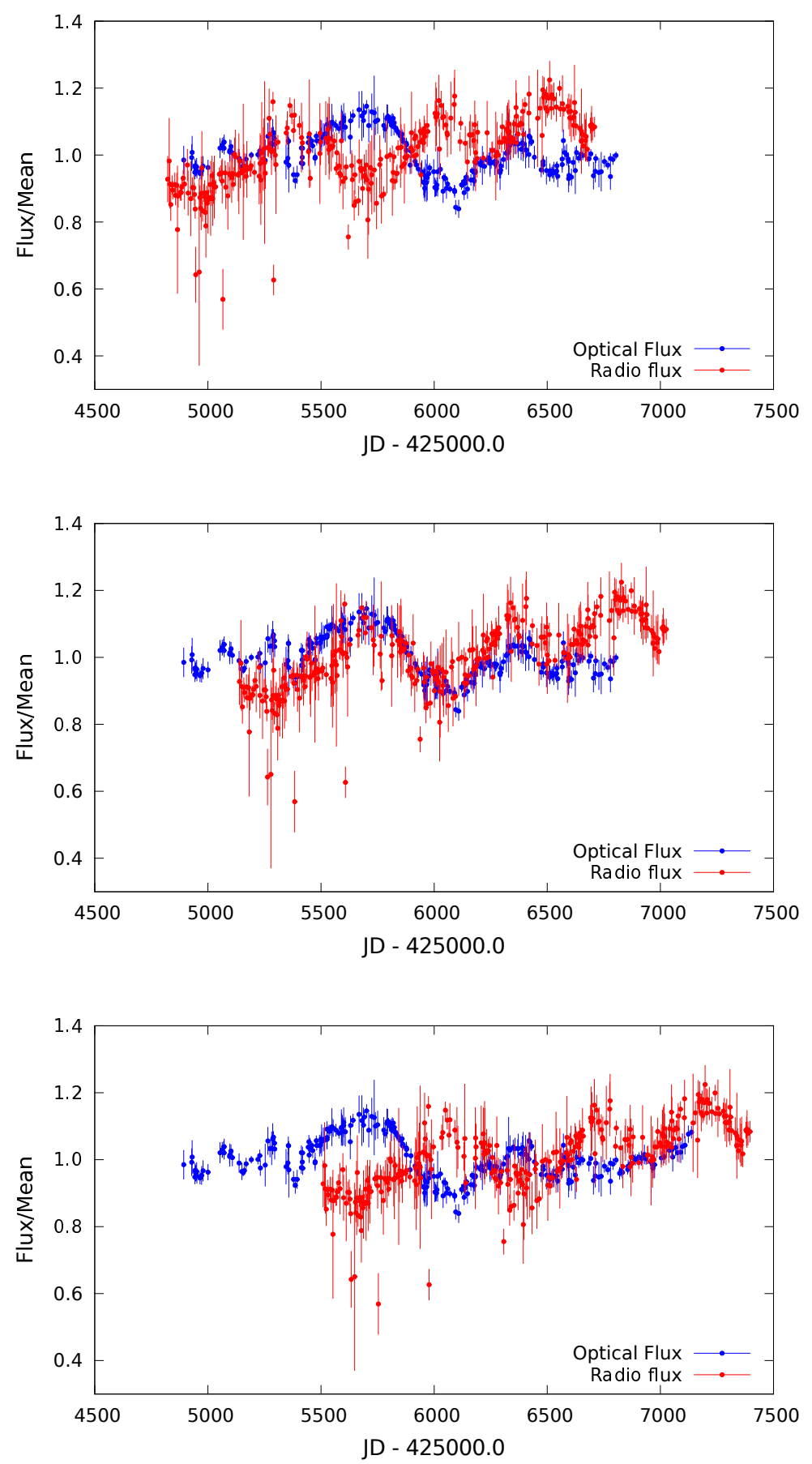

Figure 6: Normalized optical (blue) and radio (red) light curve of J2042+7508. Top panel: the time axis of radio light curve being shifted by -87 days. Middle panel: the time axis of radio light curve being shifted by +230 days. Bottom panel: the time axis of radio light curve being shifted by +600 days. 
consistent with predictions of the accretion disk instability model. We also cross-correlated optical with $15 \mathrm{GHz}$ radio data and optical with X-ray observations. For the latter no correlations were found, while the former revealed a correlation with time lag close to 230 days, however, it barely exceeds the $90 \%$ confidence level. An anticorrelation, well above the $90 \%$ confidence level, with the time lag of -87 days is present. Furthermore, DCF shows additional statistically significant peaks at longer time lags but we believe that with the current length of data, they can be just spurious and more firm conclusion can be only drawn in the future, when observations spanning over a significantly longer period of time become available.

\section{Acknowledgments}

This research has made use of data from the OVRO 40-m monitoring program ([17]) which is supported in part by NASA grants NNX08AW31G and NNX11A043G, and NSF grants AST-0808050 and AST-1109911. The work is supported in part by Polish NSC grants DEC-2013/09/B/ST9/00599 and DEC- 2012/04/A/ST9/00083.

\section{References}

[1] Aretxaga, I., Terlevich, R., 1994, MNRAS, 269, 462

[2] Ballantyne, D. R., Fabian A. C., 2005, ApJ, 622, L97

[3] Bessell, M. S., 1990, PASP, 102, 1181

[4] Brinkmann, W., Otani, C., Wagner, S. J., Siebert, J., 1998, A\&A, 330, 67

[5] Condon, J. J., Cotton, W. D., Greisen, E. W., et al., 1998, AJ, 115, 1693

[6] Edelson, R. A., Krolik, J. H., 1988, ApJ, 333, 646

[7] Erlund, M. C., Fabian, A. C., Blundell, C. M., Moss, C., Ballantyne, D .R., 2007, MNRAS, 379,498

[8] Fanaroff, B. L., Riley, J. M., 1974, MNRAS, 167, 31

[9] Hawkins, M. R. S., 1996, MNRAS, 278, 787

[10] Kataoka, J., Stawarz, L., Takahashi, Y., et al., 2011, ApJ, 740, 29

[11] Kawaguchi, T., Mineshige, S., Umemura, M., et al., 1998, ApJ, 504, 671

[12] Konar, C., Saikia, D. J., Ishwara-Chandra, C. H., Kulkarni, V. K., 2004, MNRAS, 355, 845

[13] Krimm, H. A., Holland, S. T., Corbet, R. H. D., et al., 2013, ApJSS, 209, 14

[14] Kuzmicz, A., Jamrozy, M., 2012, MNRAS, 426, 851

[15] Larsson, J., Fabian, A. C., Ballantyne, D. R., Miniutti, G., 2008, MNRAS, 388, 1037

[16] Pearson, T. J., Blundell, K. M., Riley, J. M., Warner P. J., 1992, MNRAS, 259, 13

[17] Richards, J. L., Max-Moerbeck, W., Pavlidou, V., et al., 2011, ApJS, 194, 29

[18] Riley, J. M., Warner, P. J., Rawlings, S., Saunders, R., Pooley, G. G., 1988, MNRAS, 236, 13

[19] Riley, J. M., Warner, P. J., 1990, MNRAS, 246, 1

[20] Robinson, A., Corbett, E. A., Axon, D. J., Young, S., 1999, MNRAS, 305, 97

[21] Saripalli, L., Mack, K.-H., Klein, U., Strom, R., Singal, A. K., 1996, 306, 708 
[22] Vanden Berk, D. E., Wilhite, B. C., Kron, R. G., et al., 2004, ApJ, 601, 692

[23] Welsh, W. F., 1999, PASP, 111, 1347

[24] Woo, J. H., Urry, C. M., 2002, ApJ, 579, 530

[25] Zola, S., Kuzmicz, A., Jamrozy, M., et al., ASInC, 2012, 7, 239 